\title{
Record-breaking events during the compressive failure of porous materials
}

\author{
Gergő Pál, ${ }^{1}$ Frank Raischel, ${ }^{1}$ Sabine Lennartz-Sassinek, ${ }^{2,3}$ Ferenc Kun,,${ }^{1,}$ and Ian G. Main ${ }^{2}$ \\ ${ }^{1}$ Department of Theoretical Physics, University of Debrecen, P.O. Box 5, H-4010 Debrecen, Hungary \\ ${ }^{2}$ School of Geosciences, University of Edinburgh, EH9 3FE Edinburgh, United Kingdom \\ ${ }^{3}$ Institute for Geophysics and Meteorology, University of Cologne, Cologne, Germany \\ (Received 2 July 2015; revised manuscript received 5 February 2016; published 31 March 2016)
}

\begin{abstract}
An accurate understanding of the interplay between random and deterministic processes in generating extreme events is of critical importance in many fields, from forecasting extreme meteorological events to the catastrophic failure of materials and in the Earth. Here we investigate the statistics of record-breaking events in the time series of crackling noise generated by local rupture events during the compressive failure of porous materials. The events are generated by computer simulations of the uniaxial compression of cylindrical samples in a discrete element model of sedimentary rocks that closely resemble those of real experiments. The number of records grows initially as a decelerating power law of the number of events, followed by an acceleration immediately prior to failure. The distribution of the size and lifetime of records are power laws with relatively low exponents. We demonstrate the existence of a characteristic record rank $k^{*}$, which separates the two regimes of the time evolution. Up to this rank deceleration occurs due to the effect of random disorder. Record breaking then accelerates towards macroscopic failure, when physical interactions leading to spatial and temporal correlations dominate the location and timing of local ruptures. The size distribution of records of different ranks has a universal form independent of the record rank. Subsequences of events that occur between consecutive records are characterized by a power-law size distribution, with an exponent which decreases as failure is approached. High-rank records are preceded by smaller events of increasing size and waiting time between consecutive events and they are followed by a relaxation process. As a reference, surrogate time series are generated by reshuffling the event times. The record statistics of the uncorrelated surrogates agrees very well with the corresponding predictions of independent identically distributed random variables, which confirms that temporal and spatial correlation in the crackling noise is responsible for the observed unique behavior. In principle the results could be used to improve forecasting of catastrophic failure events, if they can be observed reliably in real time.
\end{abstract}

DOI: 10.1103/PhysRevE.93.033006

\section{INTRODUCTION}

The compressive failure of heterogeneous materials proceeds in a series of individual bursts of energy release associated with discrete rupture events. Measuring the generated acoustic emissions is the primary source of information about the time evolution of the fracture process [1-7]. The statistical analysis of the stochastic time series of crackling noise in field data, laboratory experiments, and in computer simulations has provided a useful insight into the accumulation of damage and into the approach of the system to macroscopic failure. The ultimate challenge of the field is to find statistical signatures, which could be exploited to forecast the impending catastrophic failure [8]. For this purpose the analysis of synthetic time series of simulated fracture processes is indispensable since they allow a range of variables to be controlled and investigated independently, and allow representative sampling of underlying trends and statistical variability over a large number of trials $[9,10]$.

Recently, we have introduced a discrete element model of porous sedimentary rocks, which captures the essential ingredients of the materials' microstructure and of the dynamics of breaking $[11,12]$. The model was used to investigate the compressive failure of cylindrical samples under strain-controlled conditions. During the failure process we identify individual rupture events as correlated trails of breaking beams, which

\footnotetext{
*ferenc.kun@science.unideb.hu
}

are generated due to the gradual stress redistribution in the sample following local failure events [11,12].

In the present paper we investigate the internal structure of the time series of events by analyzing the statistical features of record-breaking (RB) events. Records are noise that have a size greater than any previous events of the series. Recently, the record statistics of stochastic time series has attracted great attention due to its relevance for climate and earthquake research [13-16]. Interesting analytic results have been obtained for the RB statistics of the sequences of independent identically distributed (IID) random variables for a randomly sampled stationary process [16-18]. In physics the statistics of records has been applied to understand correlated processes emerging in various types of random walks [19-21], superconductors [22], domain wall dynamics in spin glasses [23], and in chaotic processes [24]. The record statistics of crackling noise have also been studied recently in models exhibiting self organized criticality (SOC) [18] and in a mean-field model of fracture [25]. For earthquakes record statistics were recently applied to reveal spatiotemporal clustering of seismicity either by focusing on the interevent times [16] or using both the spatial and temporal distance of events $[14,15]$. In our realistic discrete element model of compressive failure we analyze both the aggregated statistics of records and the evolution of record breaking as the sample approaches macroscopic failure. As a null hypothesis we compare our results to the IID findings and to the record statistics of a surrogate data set where correlations are destroyed by randomly reshuffling all of the rupture events with respect to their origin time. This comparison makes it 
possible to reveal interesting trends and correlations in the spatial and temporal signature of the crackling noise. We show significant departures associated with nonstationary processes associated with increased strain, and reveal new signatures of impending catastrophic failure in the time series associated with record-breaking events.

\section{RECORD-BREAKING EVENTS}

We study the statistics of records in a synthetic time series of rupture events generated by a discrete element model (DEM). The model has been developed recently to investigate the emergence of crackling noise during the compressive failure of cylindrical samples of porous rocks $[11,12]$. The rock sample was reconstructed on the computer by sedimenting spherical particles with a realistic size distribution. The particles are coupled by cohesive contacts represented by beam elements, which break when they are stressed beyond a limit. Straincontrolled uniaxial compression was realized by clamping a few particle layers at the bottom and at the top of the sample. The bottom was fixed, while the top layers were moved at a constant speed along the cylinder axis. The loading process was stopped when the force acting on the top layer dropped down to zero. Due to the subsequent load redistribution following failure events beams break in cascades analogous to crackling avalanches in real materials. In Refs. [11,12] we investigated the dynamics of emergence and statistics of such crackling noise during the strain-controlled uniaxial compression of cylindrical samples composed of 20000 particles. The modeling approach proved to be successful in reproducing several important observed features of crackling noise in porous materials [1-3,6,7,26].

Figure 1 demonstrates the breaking sequence of a single simulation of a system of 20000 particles where 1832 events are obtained up to macroscopic failure in comparison with data from a real experiment [27]. In the example the breaking process starts at the deformation $\varepsilon \approx 0.0019$ where initially small events occur with size $\Delta=1$. As loading proceeds larger and larger events are triggered so that the average events size steadily increases towards failure although $\Delta$ has strong fluctuations due to the disordered microstructure of the material.

Record-breaking events are simply events that have a size $\Delta$ greater than any previous event. RB events are identified by their rank $k=1,2, \ldots$, which occurred as the $n_{k}$ th event of the complete time series with size $\Delta_{r}^{k}$. By definition the first event $n=1$ of the series is a record so that $n_{1}=1$ holds. Figure 1 illustrates that RB events form a monotonically increasing subsequence and divide the time series into segments of varying number of smaller size events. The presence of RB events has a complex effect on the local structure of the time series in Fig. 1: the moving average of the event size tends to peak at the time of record-breaking events, with a precursory increase in the average events size, followed by a decrease or relaxation after the record-breaking event. This pattern is more pronounced close to failure.

To characterize how record breaking evolves during the loading process, we also consider the size increments $\delta_{r}^{k}$ and the waiting times $m_{k}$ between consecutive records
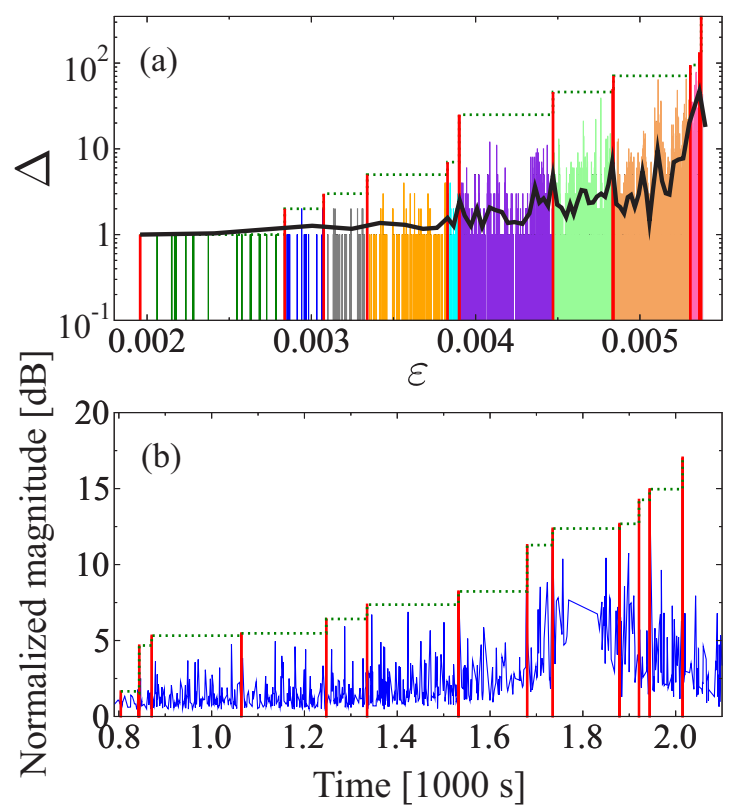

FIG. 1. (a) Sequence of events in a single simulation, i.e., the size of events $\Delta$ as a function of the strain $\varepsilon$ where they occurred. Recordbreaking events are highlighted by red bars, while subsequences between records have randomly assigned colors. The bold continuous line represents the moving average of event sizes using 30 consecutive events. For clarity, a relatively small system of 20000 particles was considered, where $n_{\max }=1832$ event occurred with 10 records. (b) Record events in a time series of a real fracture experiment performed under conditions similar to the simulations [27].

defined as

$$
\begin{gathered}
\delta_{r}^{k}=\Delta_{r}^{k+1}-\Delta_{r}^{k}, \\
m_{k}=n_{k+1}-n_{k},
\end{gathered}
$$

respectively. After analyzing the overall statistics of record quantities, we focus on the evolution of the sequence of RB events as the system approaches failure. Finally, we study how records influence the structure of the time series of crackling events.

Based on the statistics of extremes it has been shown for sequences of independent identically distributed (IID) random variables that the statistics of record-breaking events have universal features, i.e., several statistical measures of IID records do not depend on the underlying probability distribution of individual events [17]. The increasing average event size and decreasing waiting time between consecutive events in Fig. 1 demonstrate that the time series of crackling noise accompanying compressive failure is highly nonstationary. Comparing the results of our analysis to the corresponding results of IIDs enables us to quantify the competing role of the structural disorder of the material and of the stress enhancements around failed regions, which favorably lead to a stationary sequence of uncorrelated events [3,28-30] and to an accelerating sequence with spatial and temporal correlations [3,11,12,29,30], respectively. Additionally, we generate a surrogate data set by reshuffling the events of the simulated time series to destroy correlations. For each fracture 
simulation the indices $n$ of events are permutated and then the same analysis was performed as for the original data. When it is applicable, the results of the original data, its shuffled counterpart, and the IID predictions are presented together in the figures. For the data evaluations the statistical toolbox of MATLAB was extensively used [31] where a nonlinear least-square method was used for curve fitting.

In the present study careful parallelization of the computer code allowed us to substantially increase the system size. Here simulations were performed with a particle number fluctuating around $10^{5}$ such that on the average the diameter of the cylinder is spanned by about 50 particles while the height to diameter aspect ratio of the sample is 2.3 [11,12]. For an average particle size of $200 \mu$ (typical reservoir sandstone) the sample diameter would correspond to $1 \mathrm{~cm}$, which gets near to the typical small core laboratory sample diameter of $2.5 \mathrm{~cm}$. In a single simulation we identified $n_{\max }=3500-4000$ events. Averages of all quantities are calculated over 550 simulations.

\section{NUMBER OF RECORDS}

Since records are major events in the breaking process, which have a dominating contribution to the accumulating damage, it is of high interest how the number of records $N_{n}$ increases with the number of events $n$. For IIDs it has been shown that the average number of records $\left\langle N_{n}\right\rangle$ that occurred until the event number $n$ has been reached grows logarithmically with $n$

$$
\left\langle N_{n}\right\rangle \sim \kappa+\ln n+\mathcal{O}(1 / n), \quad \text { for } \quad n \rightarrow+\infty,
$$

independently of the specific form of the probability density of the random variables [17]. Here $\kappa$ denotes the EulerMascheroni constant $\kappa \approx 0.577215665[17,18]$. For crackling noise accompanying the quasistatic loading of heterogeneous materials deviations from this behavior can be expected: due to the increase of the externally imposed strain the failure process accelerates so that larger events are triggered, which follow each other after shorter waiting times $[11,12]$. The complex redistribution of stress inside the damaged sample gives rise to the emergence of temporal and spatial correlations of events in the sequence. As a consequence, the number of records increases as a power law of the event number

$$
\left\langle N_{n}\right\rangle \sim n^{\alpha}
$$

over a broad range of $n$ (see Fig. 2). The value of the exponent was obtained numerically $\alpha=0.33 \pm 0.03$. This value of $\alpha$ indicates that the number of record-breaking events also increases at a decelerating rate, albeit with a different form to that of Eq. (3). Deviations from the power law can be observed at the very beginning of the loading process and in the close vicinity of macroscopic failure. The upturn of the $\left\langle N_{n}\right\rangle$ curve in Fig. 2 for the highest event indices $n$ shows that as failure is approached the rate of record breaking accelerates (the local slope increases). This acceleration generally occurs when the record number $N_{n}$ exceeds 7 . The inset of Fig. 2 presents $\left\langle N_{n}\right\rangle$ on a semilogarithmic plot. Strong deviation can be observed from a straight line which confirms that the functional form is not logarithmic. The corresponding curve of the shuffled data is also presented in the inset, which has a perfect agreement with the analytical IID result Eq. (3). From Fig. 2 (inset) the

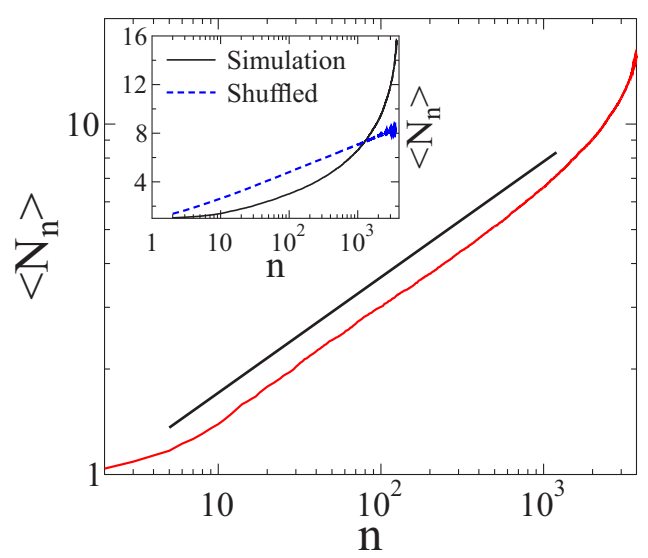

FIG. 2. Average number of records $\left\langle N_{n}\right\rangle$ occurred until the $n$th event as a function of $n$ on a double logarithmic plot. Power-law behavior is obtained over a broad range of $n$. The straight line represents a power law of exponent 0.33 . The inset presents the same quantity $\left\langle N_{n}\right\rangle$ on a semilogarithmic plot. For comparison the shuffled data set is also included where a logarithmic dependence is found.

null hypothesis of sampling from a parent IID distribution can be rejected. Instead we have a decelerating transient response of a power-law form.

To characterize the sample-to-sample variation of the number of records $N_{n}$ we calculated the standard deviation

$$
\operatorname{Var}\left(N_{n}\right)=\left\langle\left(N_{n}-\left\langle N_{n}\right\rangle\right)^{2}\right\rangle .
$$

For IIDs the analytic solution gives again an asymptotic logarithmic increase with $n$ as $[17,18]$

$\operatorname{Var}\left(N_{n}\right)=\kappa+\ln n-\frac{\pi^{2}}{6}+\mathcal{O}(1 / n)$, for $n \rightarrow+\infty$,

so that the relative variance $\operatorname{Var}\left(N_{n}\right) /\left\langle N_{n}\right\rangle$ monotonically increases and tends to 1 for sufficiently large $n$. Figure 3 shows that the very beginning of our fracture process, up to about

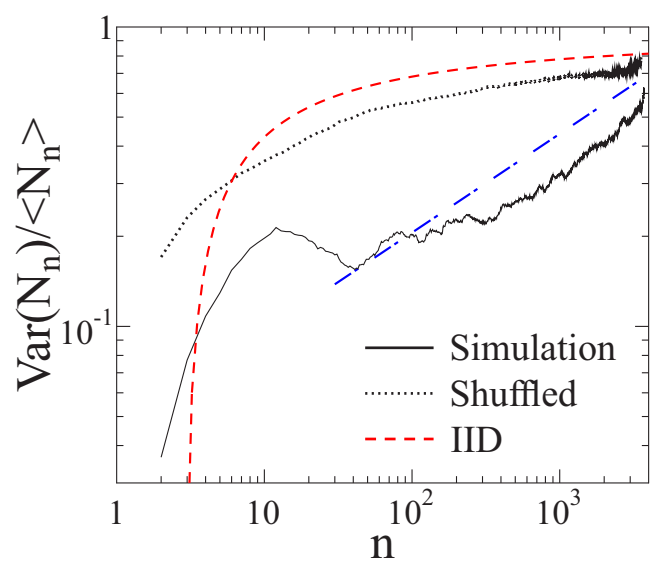

FIG. 3. Standard deviation of the number of records $\operatorname{Var}\left(N_{n}\right)$ occurred until the $n$th event divided by the average record number $\left\langle N_{n}\right\rangle$ on a double logarithmic plot. The red (dashed) and the blue (dashed-dotted) lines represent the corresponding result of the shuffled data set and a power law of exponent $1 / 3$, respectively. 


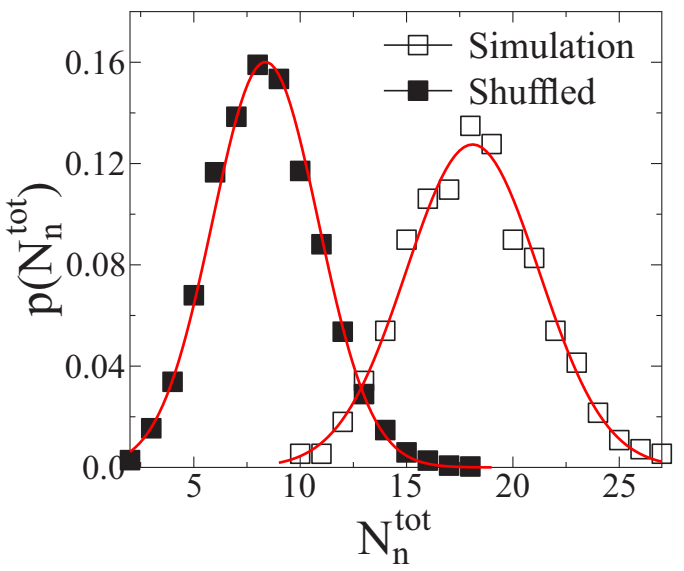

FIG. 4. Probability distribution of the total number of records $p\left(N_{n}^{\text {tot }}\right)$ that occurred up to failure for the original and surrogate data sets. The continuous lines represent the Gaussian distributions obtained with the mean and standard deviation calculated directly from the data.

$n=10$ events, is consistent with the IID behavior. However, after a short intermediate decreasing regime the curve sets to a faster increase, which has a nearly power-law functional form. In Fig. 3 a power law of the same exponent as for the average record number in Fig. 2 is drawn to guide the eye. The result of the shuffled data is again consistent with the IID solution.

The probability distribution of the number of records $p\left(N_{n}\right)$ that occurred up to a fixed number $n$ of events has been found to have a Gaussian functional form for IIDs in the limit of large $n$ values [17,18]. Figure 4 shows that in our fracture process the distribution $p\left(N_{n}^{\text {tot }}\right)$ of the total number of records $N_{n}^{\text {tot }}$ that occurred up to failure is similar to a Gaussian with an average $\left\langle N_{n}^{\text {tot }}\right\rangle=18.1$ and standard deviation $\operatorname{Var}\left(N_{n}^{\text {tot }}\right)=3.1$. The highest and lowest number of records we identified in single simulations were 10 and 26 , respectively, with the most probable value 18 . In the shuffled event series large-size events can occur anywhere, which results in a significantly lower number of records $\left\langle N_{n}^{\text {tot }}\right\rangle=8.4$ with a standard deviation $\operatorname{Var}\left(N_{n}^{\text {tot }}\right)=2.5$. The continuous lines in Fig. 4 represent Gaussian distributions obtained by inserting the corresponding values of $\left\langle N_{n}^{\text {tot }}\right\rangle$ and $\operatorname{Var}\left(N_{n}^{\text {tot }}\right)$ for the two data sets. Both for the simulated and surrogate data reasonable agreement is obtained with the Gaussian distribution.

\section{DISTRIBUTION OF RECORD SIZES}

Recently, we have shown that the size distribution of events $p(\Delta)$ accumulating all events up to failure during the compression process has a power-law functional form followed by an exponential cutoff

$$
p(\Delta) \sim \Delta^{-\xi} \exp \left[-\left(\Delta / \Delta^{*}\right)^{c}\right] .
$$

In Refs. [11,12] the exponent $\xi$ was obtained numerically as $\xi=2.15$ for samples comprising 20000 particles. In the present study $10^{5}$ particles are used, which gives the same exponent for $p(\Delta)$, however, with a broader power-law regime in Fig. 5. Thus, there is no evidence of scale dependence in the

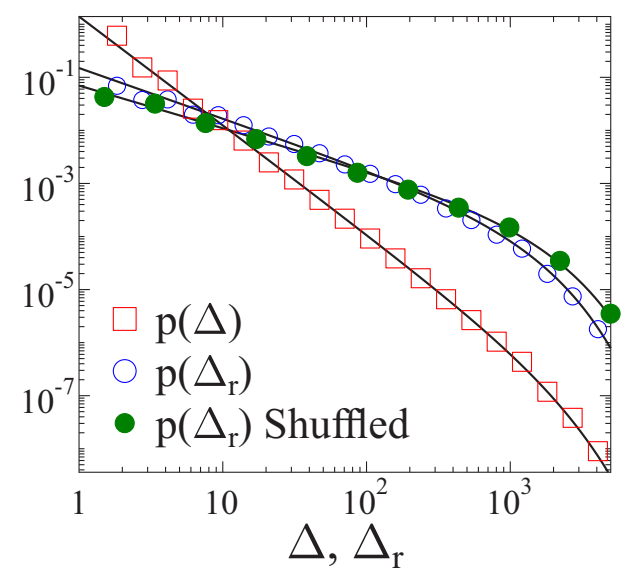

FIG. 5. Probability distribution $p(\Delta)$ of the event size $\Delta$ considering all events up to failure. The size distribution of records $p\left(\Delta_{r}\right)$ is presented both for the original and surrogate data sets. In all cases the bold lines represent best fits with Eq. (7) obtained with the parameter values: $\xi=2.15, c=1.0$ and $\Delta^{*}=1810$ for $p(\Delta)$, $\xi_{r}=1.0, c_{r}=0.9$ and $\Delta_{r}^{*}=1052$ for $p\left(\Delta_{r}\right)$, and $\xi_{r}^{s}=0.8, c_{s}=0.9$ and $\Delta_{s}^{*}=1270$ for for shuffled series.

power-lay exponent, only an increase in the characteristic size $\Delta^{*}$, and hence in the bandwidth of the power-law behavior.

Records form a subset of events of the complete series containing solely the largest events that occurred up to a given event index. Figure 5 shows that the distribution of the size of records $p\left(\Delta_{r}\right)$ has the same functional form Eq. (7) as the size distribution of all events $p(\Delta)$, however, the exponent $\xi_{r}$ of the power-law regime is significantly lower $\xi_{r}=1.0 \pm 0.05$ than for the complete distribution $\xi$. Selecting RB events implies a resampling of the ensemble of events, where the distribution of the record size is governed by the statistics of extremes. The difference in slope is likely caused by the inherent sample bias in choosing record-breaking events either (i) because this preferentially filters out smaller events and/or (ii) larger events may be associated with a greater degree of local stress concentration (stress intensity), known to be associated with a flatter slope [1]. In the shuffled event series large-size events can occur earlier than during the original fracture process so that small-size events have a lower chance to become a record. As a consequence, the size distribution of shuffled records has the same functional form, however, with a lower exponent $\xi_{r}^{s}=0.8 \pm 0.07$, which indicates the lower fraction of small records.

\section{APPROACH TO FAILURE THROUGH BREAKING OF RECORDS}

The integrated statistics of records presented above gives an overall description of the subset of RB events of the crackling time series. It is a question of fundamental interest how the system approaches failure through a sequence of record breaking events. To obtain a quantitative understanding we calculated averages of characteristic quantities of records as a function of the record rank $k$. Figure 6(a) shows that as the system evolves the average size of records $\left\langle\Delta_{r}^{k}\right\rangle$ rapidly increases with $k$. The size increments $\left\langle\delta_{r}^{k}\right\rangle$ exhibit qualitatively the same behavior, i.e., during the entire failure process records 

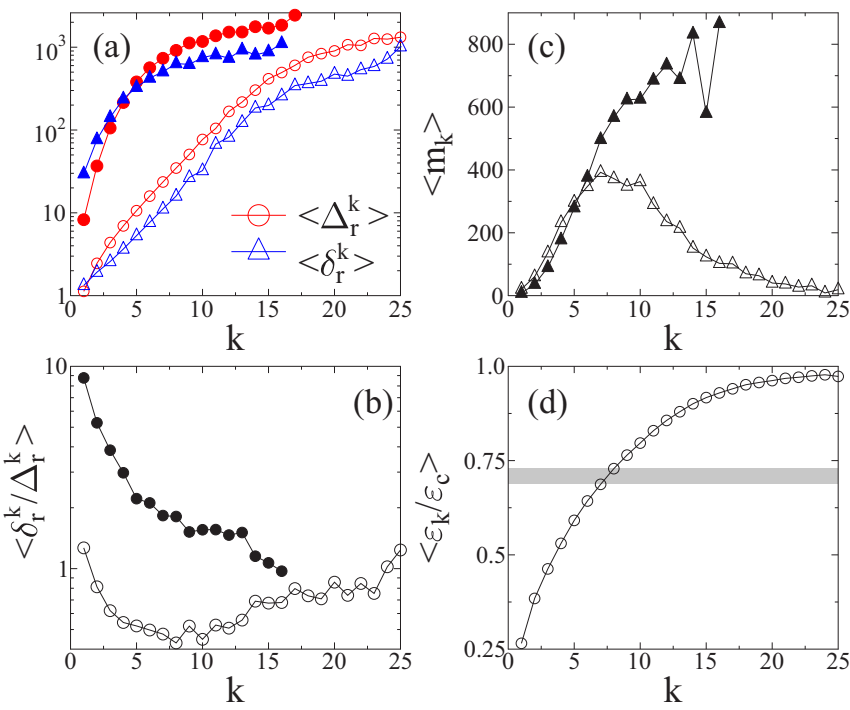

FIG. 6. Average value of the characteristic quantities of records as a function of the record rank $k$ : (a) size and increment, (b) relative increment, (c) waiting time, and (d) strain of records normalized by the critical strain of failure. The gray stripe highlights the strain range between the records of rank $k=7$ and $k=8$. In (a), (b), (c) the open and filled symbols represent the original and the surrogate data sets, respectively.

get broken with an increasing sequence of increments. The shuffled data set has the same qualitative tendencies with the difference that low rank records reach higher sizes than in the original data.

For a stationary sequence of IIDs the extreme value statistics leads to a monotonous decrease of average relative increments between consecutive records [32]. This is what we find for the early part of our time series, when the timing and size of events is dominated by the random structural disorder. However, for the later RB events we find the opposite, i.e., an accelerating trend, most likely associated with processes dominated by the dynamics, such as stress relaxation and redistribution, and localization of events on to the eventual failure plane. This change from deceleration to acceleration is illustrated in Fig. 6(b) where the average value of the relative increment $\left\langle\delta_{r}^{k} / \Delta_{r}^{k}\right\rangle$ is presented. Initially the RB process slows down, i.e., up to a characteristic record rank $k^{*}=7-8$ the relative increment $\left\langle\delta_{r}^{k} / \Delta_{r}^{k}\right\rangle$ decreases, while for higher ranks $k>k^{*}$ the onset of accelerating fracture is marked by the increase of $\left\langle\delta_{r}^{k} / \Delta_{r}^{k}\right\rangle$. The result is also supported by its absence in the surrogate data where correlation are destroyed: the relative increment monotonically decreases without any sign of qualitative change.

After a record has occurred as the $n_{k}$ th event of the sequence it gets broken by the next record, which is the $n_{k+1}$ th event of the evolving system. The waiting time $m_{k}=n_{k+1}-n_{k}$ is an important characteristic of the dynamics; it provides the number of events one has to wait to break the $k$ th record by the $(k+1)$ th one. The quantity $m_{k}$ is the lifetime of the $k$ th record. For IIDs it has been shown analytically that the probability distribution $p(m)$ of waiting times $m$ has a power-law behavior

$$
p(m) \sim m^{-z}
$$

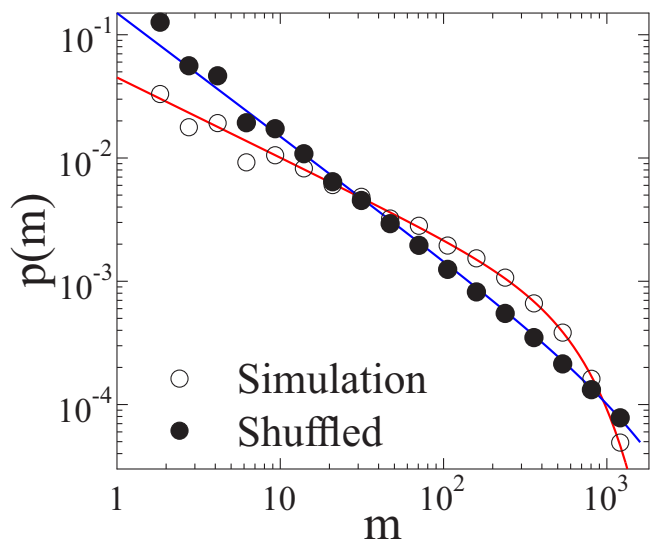

FIG. 7. Distribution $p(m)$ of waiting times $m$ between consecutive records for fracture simulations and for the surrogate data. The bold lines represent best fit, which was obtained using the generic functional form of Eq. (7). The value of the power-law exponent is $z=0.62$ and $z=0.98$ for the simulated and shuffled data, respectively.

with a universal exponent $z_{I I D}=1$. Figure 7 demonstrates that the lifetime distribution of the surrogate is consistent with the IID prediction with an exponent $z_{s}=0.98 \pm 0.07$. For the fracture process the same functional form is obtained, however, with a different exponent $z=0.62 \pm 0.03$. The low exponent $z<z_{I I D}$ reflects the fact that during compressive failure of heterogeneous materials long waiting times between records occur more frequently than for a random sequence of IIDs. Since record breaking is controlled by the statistics of extremes, in a sequence of IIDs waiting times get larger with the record rank $k$, since it takes longer to break a larger record. However, in the fracture process progressively longer waiting times occur at the beginning. In spite of their larger size, records of higher rank may get broken faster because of the rapid increase of event size when approaching failure.

The emergence of a characteristic record rank $k^{*}$ is further supported by the behavior of the average value of waiting times $m_{k}$. Figure $6(\mathrm{c})$ presents the remarkable result that the record rank $k^{*}=7$ separates two qualitatively different regimes of the time evolution of the compressed system: at the beginning of the failure process $k<k^{*}$ the increasing waiting time implies a deceleration of record breaking, where it takes longer and longer to break the growing records. For IIDs the average waiting time must be monotonically increasing [18] as observed in Fig. 6(c) for the surrogate data set since records can be overcome after a larger and larger number of trials drawn from the same distribution. Comparing our simulation results to the IID findings, the increasing regime of $\left\langle m_{k}\right\rangle$ can be attributed to the dominance of disorder in the fracture process consistent with our earlier findings $[11,12]$. Beyond $k^{*}$ the waiting time starts to decrease confirming the change of the dynamics. In spite of their larger size, records get broken after fewer and fewer small sized events, which indicate the dominance of temporal and spatial correlations in triggering consecutive events.

In Ref. [11] our simulations revealed the localization of individual rupture events to a damage band, which occurs at a characteristic strain value close to failure. Such 


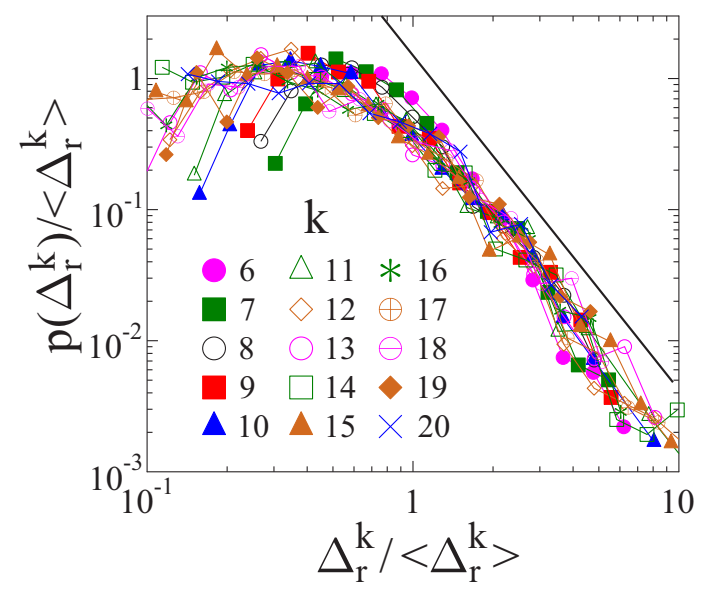

FIG. 8. Probability distribution $p\left(\Delta_{r}^{k}\right)$ of record sizes $\Delta_{r}^{k}$ for fixed ranks $k$ rescaled with the average record size presented in Fig. 6(a). Good quality collapse is obtained.

localization has also been seen in acoustic emission data during laboratory experiments loaded at constant strain rate [27]. The spatial localization is accompanied by a rapid increase of the average event size [11,12]. In Fig. 6(d) the average strain $\left\langle\varepsilon_{k} / \varepsilon_{c}\right\rangle$ where the records occurred is presented normalized by the critical strain of macroscopic failure. The strain range $\left\langle\varepsilon_{k} / \varepsilon_{c}\right\rangle \approx 0.69-72$ corresponding to the record ranks $k^{*}=7-8$ is also highlighted in the figure. Comparing to the strain of localization $\left\langle\varepsilon_{k} / \varepsilon_{c}\right\rangle \approx 0.85-0.9$ it follows that the acceleration of the RB process sets in significantly earlier. The macroscopic response of the system proved to be quasi-brittle, i.e., linearly elastic behavior is obtained where stronger nonlinearity emerges solely close to failure due to the intense nature of the crackling noise $[11,12]$. The yield stress $\sigma_{Y}$ and the corresponding strain $\varepsilon_{Y}$, which mark the onset of nonlinearity of the constitutive curve $\sigma(\varepsilon)$, could be identified by computer simulations as $\sigma_{Y} / \sigma_{c} \approx 0.73$ and $\varepsilon_{Y} / \varepsilon_{c} \approx 0.7$, respectively. The characteristic strain $\left\langle\varepsilon_{k^{*}} / \varepsilon_{c}\right\rangle$ of accelerated record breaking falls close to $\varepsilon_{Y}$.

To get a more detailed characterization of the evolution of the system towards failure, we evaluated the size distribution of records for fixed ranks $p\left(\Delta_{r}^{k}\right)$. Figure 8 presents a scaling plot where distributions of different record ranks are rescaled with the average record size $\left\langle\Delta_{r}^{k}\right\rangle$ presented in Fig. 6(a). Except for the first few bins of the smallest record sizes good quality data collapse is obtained, which implies the validity of the scaling structure

$$
p\left(\Delta_{r}^{k}\right)=\left\langle\Delta_{r}^{k}\right\rangle \Phi\left(\Delta_{r}^{k} /\left\langle\Delta_{r}^{k}\right\rangle\right),
$$

where $\Phi(x)$ denotes the scaling function. The result demonstrates that records of different ranks have the same size distribution, they get only shifted to accommodate the increasing average size. Note that the functional form of the scaling function $\Phi(x)$ can be approximated as a power law for $x>1$. The slope of the straight line in Fig. 8 is 2.55. In the scaling analysis records of the lowest and highest ranks are not included because their size spans only a narrow range, and we do not have a sufficient number of events, respectively. When the event series is shuffled, any event can be the first, and hence, the first record. However, for higher ranks small

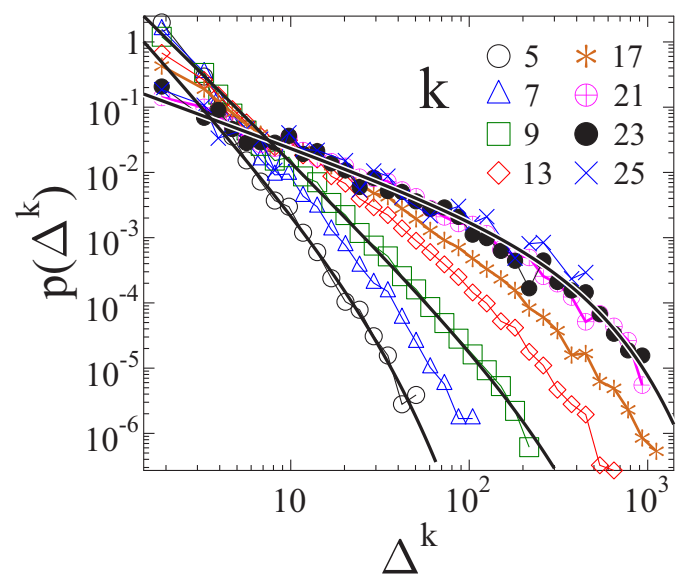

FIG. 9. Probability distribution $p\left(\Delta^{k}\right)$ of event sizes in subsequences of events between consecutive records. For demonstration three curves were fitted with the functional form Eq. (10). For the highest ranks $k \geqslant 20$ the distributions practically fall on top of each other.

size events have a very low chance to become a record. As a consequence the surrogate data set cannot have such a scaling behavior because the functional form of the size distribution changes with the record rank: the size distribution of the first record is practically identical with the complete size distribution of events (see Fig. 5), while for higher record ranks the distribution must have a slower decay.

In Fig. 1 it has been highlighted that consecutive records enclose subsequences of the complete time series. To explore this further we analyzed how the size distribution $p\left(\Delta^{k}\right)$ of these subsequences between the $k$ th and $(k+1)$ th records evolve as the system approaches failure. We excluded the two record events at the left- and right-hand sides of the sub-sequence from the statistics. Figure 9 presents the resulting size distributions $p\left(\Delta^{k}\right)$ for several $k$ except for the lowest ranks $(k<5)$ where event sizes span only a very narrow region so that no meaningful distributions could be obtained. The distributions cannot be collapsed by rescaling with the average, only the cutoff of the distributions could be scaled on the top of each other. The reason is that the distributions have a power-law functional form followed by an exponential cutoff,

$$
p\left(\Delta^{k}\right) \sim\left(\Delta^{k}\right)^{-\tau_{k}} e^{-\Delta^{k} / \Delta_{s}^{k}},
$$

however, the power-law exponents $\tau_{k}$ are different for different record ranks $k$. It can also be observed in Fig. 9 that the cutoff scale $\Delta_{s}^{k}$ grows with the record rank $k$ and the curves of the highest ranks $k \geqslant 20$ fall practically on top of each other. Figure 10 presents the exponent $\tau_{k}$ obtained by fitting with Eq. (10). For the lowest rank the exponent has a high value $\tau_{k}=3.26$ then it monotonically decreases and for the highest ranks it tends to the vicinity of $\tau_{k}=1.0$.

The result is an interesting manifestation of the $b$-value anomaly [33] (i.e., the change in the exponent of the loglinear frequency-magnitude distribution) we have pointed out before in DEM simulations of compressive failure [11]. In subsequences between records the number of events is practically equal to the waiting time $m_{k}$, which covers a broad range [see Fig. 6(b)]. In the traditional analysis of the time 


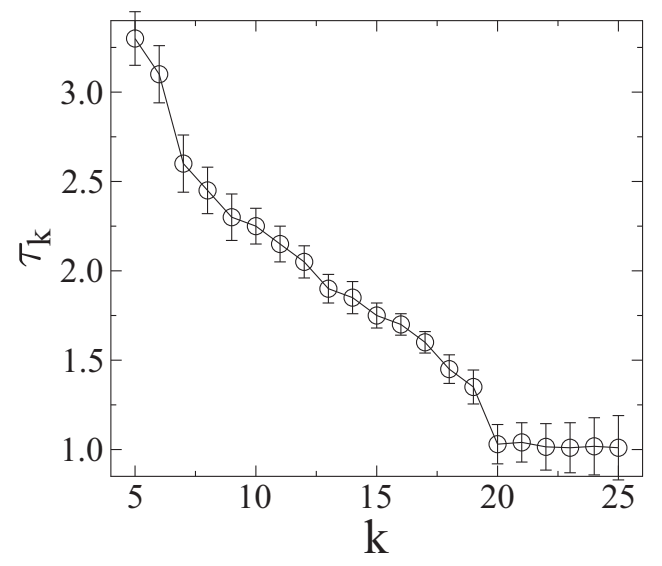

FIG. 10. The value of the exponent $\tau_{k}$ of the size distribution of subsequences of events between consecutive records as a function of the record rank $k$, together with the error bars.

series of events, event windows are considered with a fixed number (typically a few hundred) of events. This has the consequence that windows close to failure involve more and more records and subsequences, which results in an exponent different from the one of single subsequences presented above [11,12].

Figure 11 shows that no such behavior exists when correlations are destroyed by shuffling the event series. Except for the lowest ranks, practically all the distributions of the subsequences fall on the top of each other. The emerging master curve can be well fitted with the functional form Eq. (10) where the exponent takes the value $\tau=2.1$ falling close to the size distribution exponent of the whole population of the events. The result is reasonable since all the subsequences are random samples of the complete event set.

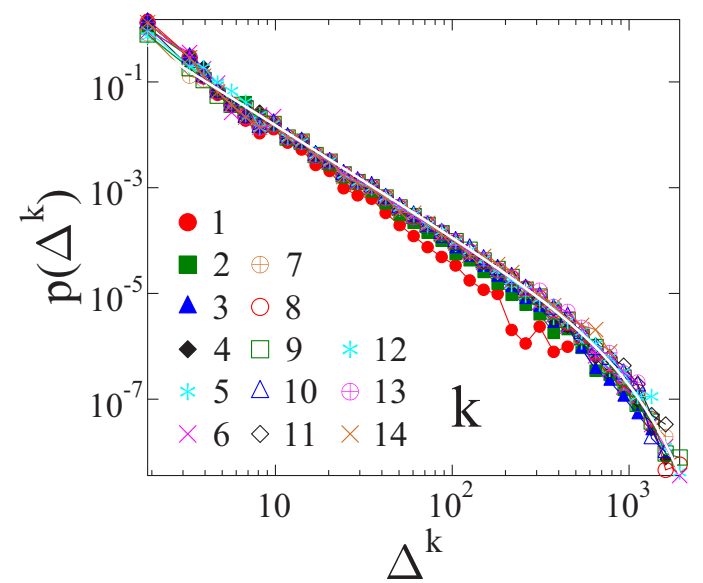

FIG. 11. Probability distribution $p\left(\Delta^{k}\right)$ of event sizes in subsequences of the shuffled event series between consecutive records. Except for the lowest ranks the curves fall on the top of each other. The white curve was obtained by fitting the functional form Eq. (10) with an exponent $\tau=2.1$ nearly equal to the one of the size distribution including all the events.

\section{STRUCTURE OF THE TIME SERIES}

Records are major events of the breaking activity of the compressed sample, which can have a strong effect on both the spatial and temporal occurrence of subsequent events. In Fig. 1 the increasing value of the moving average of the event size towards records may indicate some kind of precursory activity preceding record-breaking events. In order to obtain a more detailed description of the internal structure of the time series we calculated the average size of events $\left\langle\Delta_{n}\right\rangle$ before and after records of different ranks $k$ as a function of the event index $n-n_{k}$ relative to the records $n_{k}$. In Fig. 12 zero index corresponds to the records while positive and negative values of $n-n_{k}$ stand for events preceding and following records, respectively. For clarity, the $\left\langle\Delta_{n}\right\rangle$ curves are normalized by the average size $\left\langle\Delta_{r}^{k}\right\rangle$ of the corresponding record.

It can be observed in Fig. 12 that low rank records just randomly pop up on a flat background without any signature of the imminent record event. However, high rank records are approached through a sequence of precursory events with an increasing average event size, and they are followed by a relaxation process characterized by a gradually decreasing event size. In this sense the later record-breaking events mimic the behavior of the final dynamic rupture event. The functional form of $\left\langle\Delta_{n}\right\rangle$ can be well fitted by an inverse power law

$$
\left\langle\Delta_{n}\right\rangle \sim\left|n-n_{k}\right|^{-\gamma}
$$

on both sides of high rank records. In Fig. 12 a best fit is obtained with the exponents $\gamma=0.38$ and $\gamma=0.31$ before and after records, respectively, considering the curves of $k=18,20$.

To characterize the temporal occurrence of events we analyzed in a similar way the average value of the physical waiting time $\left\langle T_{n}\right\rangle$ between consecutive events before and after records as a function of the event index $n-n_{k}$ relative to record indices $n_{k}$. Figure 13 shows that for low record ranks $k<7$ the $\left\langle T_{n}\right\rangle$ curves have a sharp local minimum at records, which implies that the records are approached by a short

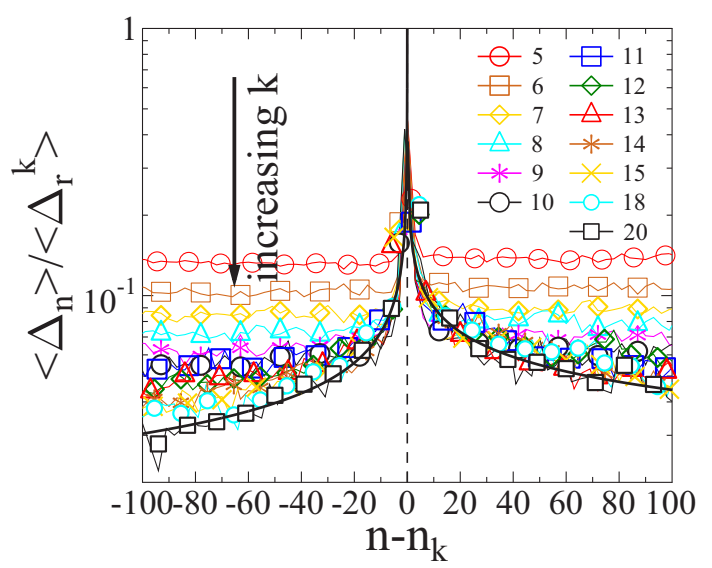

FIG. 12. Average size of events $\left\langle\Delta_{n}\right\rangle$ in the vicinity of records as a function of their integer index relative to records $n-n_{k}$. High rank records are preceded by events of increasing size and they are followed by a relaxation process where the event size gradually decreases. The bold line represents fit with Eq. (11). 


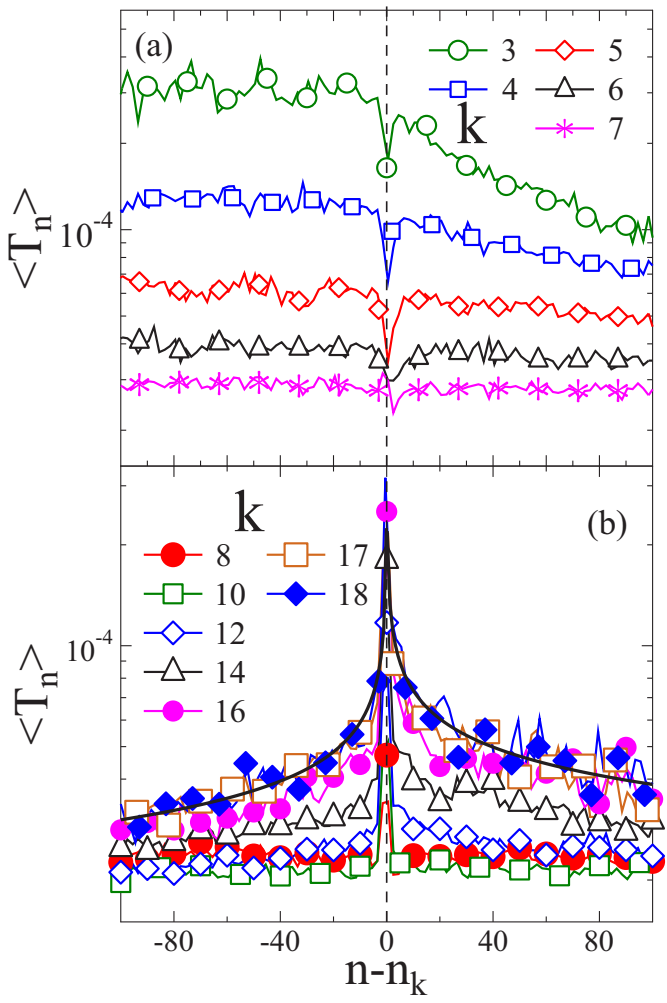

FIG. 13. Average value of the physical waiting time $\left\langle T_{n}\right\rangle$ between consecutive events as a function of the event index relative to records $n-n_{k}$. The low and high rank records are presented separately in (a) and (b), respectively. The red bold continuous lines in (b) represent fit with Eq. (11).

period of increasing event rate and they are followed by a few relaxation events with increasing waiting times in between. However, for higher record ranks $k \geqslant 7$ the overall behavior drastically changes: The $\left\langle T_{n}\right\rangle$ curves develop a broader and broader maximum at records, which shows that large-size events occurring in the vicinity of records (compare also to Fig. 12) are followed by longer waiting times. This behavior is the consequence of the strain-controlled loading, i.e., after large-size events it takes longer to build up again the stress field and initiate the next event. In a different context, studying the correlation of event size and the waiting time after events we have found analogous behavior in Ref. [11,12]. The present result is another form of appearance of the size-waiting-time correlation.

In the deceleration and acceleration periods the $\left\langle T_{n}\right\rangle$ curves can be well fitted with the power-law functional form. For the exponents providing best fit in Fig. 13 the same value 0.29 was obtained numerically on both sides of records.

\section{DISCUSSION}

We investigated the statistics of record-breaking events generated during the compressive failure of heterogeneous materials. A synthetic time series of crackling events was generated by discrete element simulations of a realistic model where a cylindrical sample was subject to uniaxial compression in a strain-controlled way. Records of the time series of crackling events are identified as a local dynamic rupture event with size greater than any previous event. The large system size in the model and the large number of samples generated by simulations allowed us to obtain high-quality results for the statistics of records. In order to reveal the role of correlations of events the analysis was repeated for a surrogate time series, which was generated by randomly reshuffling the simulated events. Additionally, our results were compared to the corresponding analytic findings on sequences of independent identically distributed random variables.

The overall statistics of records is characterized by powerlaw distributions of the size and lifetime of records and by the power law increase of the record number with the total number of events. This behavior deviates from the surrogate data set where correlations were destroyed, however, the later one agreed very well with the stationary process of IIDs.

As the compression proceeds the system gradually evolves towards macroscopic failure where the sample loses its integrity. We have shown earlier $[11,12]$ that the failure process has two qualitatively different stages: the beginning of the failure process is dominated by the structural disorder of the sample giving rise to an uncorrelated emergence of smallsized events. Later on as macroscopic failure is approached the fracture process accelerates, which is indicated by the increasing event size and by the decreasing waiting times between consecutive events. This second stage is dominated by the growing spatial and temporal correlations of local rupture events in the sample. In the present paper we showed that analyzing the statistics of record-breaking events and their evolution with increasing rank, provides an alternative way for the quantitative characterization of the emerging correlations in the fracture process as the loaded system evolves towards failure.

Our analysis revealed the existence of a characteristic record rank $k^{*}$, which separates two regimes of the fracture process: for low record rank $k<k^{*}$ the process of record breaking slows down, i.e., it takes longer and longer to break a record. At $k^{*}$ the $\mathrm{RB}$ process starts to accelerate indicated by the rapidly decreasing record lifetime and by the increasing relative size increments of records. The surrogate event series proved to follow the IID predictions without any sign of the emergence of a characteristic record rank.

Records are found to affect the surrounding structure of the time series: approaching high rank records, events have a gradually increasing size separated by an increasing physical waiting time, while after records the event size decreases and the temporal occurrence accelerates with decreasing waiting times. The evolution of both the event size and waiting time is characterized by power-law functional forms as a function of the distance from records. The result is consistent with our earlier finding presented in Ref. [12], where the increase of waiting time after large-size events has been pointed out by analyzing the size-waiting-time correlation of events. The reason is the strain controlled loading, which has the consequence that larger events release the stress in a larger volume of the specimen, and hence, it takes longer to build up the stress field again and trigger the next event.

Scaling analysis revealed that records of fixed ranks have the same size distribution, which has power-law asymptotics. Records split the time series of events into subsequences, 
which typically contain fewer and fewer events as the system approaches macroscopic failure. We showed that the size distribution of events in subsequences are characterized by power-law size distributions, however, the exponent spans a broad range decreasing to the vicinity of one towards failure. This result is consistent with the trend of the $b$-value anomaly of the time series [1]. In traditional $b$-value analysis of crackling time series windows of events are considered either with a fixed number of events or with a fixed strain or stress increment. Since records affect the structure of the time series, our analysis suggests that focusing on subsequences between records can provide significant additional and relevant information to that which can be inferred from the average properties of the time series of the whole population of all events. In principle this could improve forecasting power for catastrophic failure, but this depends on our being able to detect the processes revealed here in real time, and in a single realization, during a live experiment.

Our simulations were carried out with samples of about 100000 particles, which corresponds to practically laboratory scale sample sizes. Comparison to simulations of a significantly smaller system size of 20000 particles (see Refs. [11,12]) showed that in larger systems a larger number of events are generated whose size distribution spans a broader range. However, the probability distributions of the characteristic quantities of the event ensemble are quite robust, i.e., the value of the power-law exponents are the same within the error bars. The same is valid for the number and size of records, however, for the value of the characteristic record rank $k^{*}$ no size dependence could be identified. In the simulations with 100000 particles the relative fluctuations in the total number of events is quite moderate (below 0.1). Tests of the effect of these fluctuations, e.g., by restricting the analysis to simulations where the number of events exceeds a threshold, revealed that they mainly affect the statistics of the results but no systematic bias occurred.

Our analysis strongly relies on the comparison of the record statistics of crackling noise time series to the corresponding IID findings and to the results of uncorrelated surrogates. A similar strategy has recently been applied in Ref. [18] to study the avalanche dynamics of models of self-organized criticality where IID results were derived by substituting the known steady-state distribution of avalanche sizes into the generic IID formulas. Deviations of the record statistics of SOC models from the IID results were identified as signatures of correlations emerging in the dynamics of avalanches. Record breaking statistics of daily temperatures has proven a useful tool to investigate the effect of global warming. It has been pointed out in Ref. [34] that the observed frequency and average value of record temperatures, at least one locality, can be understood in terms of a stationary climate, so that the current warming rate does not have a noticeable effect on the record statistics of daily temperatures (at least in the city of Philadelphia where the data was measured). Additionally, the ratio of the number of record high (record of the highest temperature of a given day) and record low (record of the lowest temperature of a given day) temperatures was suggested as a useful measure to point out trends in time series [34]. This idea was extended to earthquakes by Ref. [16] where the sequence of interval times between successive earthquakes were analyzed. It was shown that for global earthquakes the statistics of both record-breaking longer and record-breaking shorter intervals is consistent with IID processes. This implies that record-breaking earthquakes in the global catalog are independent each other, as indeed are most main shocks. However, within an isolated aftershock sequence, where interevent times get systematically longer, the number of record-breaking longer intervals was found to increase as a power law of the event number similarly to our result Eq. (4). At the same time the ratio $r$ of the number of record-breaking longer and shorter intervals proved to be predominantly greater than one, which was suggested as a measure to distinguish between background seismicity and aftershock sequences [16]. Extending the calculation of the ratio $r$ to a broader spatiotemporal interval, before and after the main shock, $r<1$ and $r>1$ were found, respectively, which addressed a possibility of using record-breaking statistics for forecasting [16]. For interevent times of aftershocks the Omori-type relaxation was proved to be responsible for the power-law increase of the number of records, while in our case the monotonically increasing average event size towards failure plays a similar role. For earthquakes, the frequency-magnitude distributions for main shocks and aftershocks are the same, however, for fracture processes both the event magnitude and interevent time exhibit a systematic evolution towards macroscopic failure. In the present study we focused on the record-breaking process of event magnitudes, the extension to interevent times is a work in progress including also the analysis of the correlation of consecutive records [35].

\section{ACKNOWLEDGMENTS}

The work is supported by the projects TAMOP-4.2.2.A11/1/KONV-2012-0036. The project is implemented through the New Hungary Development Plan, cofinanced by the European Union, the European Social Fund, and the European Regional Development Fund. F.K. acknowledges the support of OTKA K84157.
[1] P. R. Sammonds, P. G. Meredith, and I. G. Main, Nature (London) 359, 228 (1992).

[2] I. O. Ojala, I. G. Main, and B. T. Ngwenya, Geophys. Res. Lett. 31, L24617 (2004).

[3] K. Mair, I. Main, and S. Elphick, J. Struct. Geol. 22, 25 (2000).

[4] J. Henderson, I. Main, P. Meredith, and P. Sammonds, J. Struct. Geol. 14, 905 (1992).
[5] J. Rosti, X. Illa, J. Koivisto, and M. J. Alava, J. Phys. D 42, 214013 (2009).

[6] G. F. Nataf, P. O. Castillo-Villa, J. Baró, X. Illa, E. Vives, A. Planes, and E. K. H. Salje, Phys. Rev. E 90, 022405 (2014).

[7] P. O. Castillo-Villa, J. Baró, A. Planes, E. K. H. Salje, P. Sellappan, W. M. Kriven, and E. Vives, J. Phys. Cond. Matt. 25, 292202 (2013).

[8] I. Main, Rev. Geophys. 34, 433 (1996). 
[9] M. Alava, P. K. Nukala, and S. Zapperi, Adv. Phys. 55, 349 (2006).

[10] R. C. Hidalgo, F. Kun, K. Kovács, and I. Pagonabarraga, Phys. Rev. E 80, 051108 (2009).

[11] F. Kun, I. Varga, S. Lennartz-Sassinek, and I. G. Main, Phys. Rev. E 88, 062207 (2013).

[12] F. Kun, I. Varga, S. Lennartz-Sassinek, and I. G. Main, Phys. Rev. Lett. 112, 065501 (2014).

[13] G. Wergen and J. Krug, Europhys. Lett. 92, 30008 (2010).

[14] J. Davidsen, P. Grassberger, and M. Paczuski, Geophys. Res. Lett. 33, 111304 (2006).

[15] J. Davidsen, P. Grassberger, and M. Paczuski, Phys. Rev. E 77, 066104 (2008).

[16] M. R. Yoder, D. L. Turcotte, and J. B. Rundle, Nonlin. Proc. Geophys. 17, 169 (2010).

[17] G. Wergen, J. Phys. A: Math. Theor. 46, 223001 (2013).

[18] R. Shcherbakov, J. Davidsen, and K. F. Tiampo, Phys. Rev. E 87, 052811 (2013).

[19] S. N. Majumdar and R. M. Ziff, Phys. Rev. Lett. 101, 050601 (2008).

[20] S. N. Majumdar, G. Schehr, and G. Wergen, J. Phys. A: Math. Theor. 45, 355002 (2012).

[21] C. Godreche, S. N. Majumdar, and G. Schehr, J. Phys. A: Mat. Theor. 47, 255001 (2014).
[22] L. P. Oliveira, H. J. Jensen, M. Nicodemi, and P. Sibani, Phys. Rev. B 71, 104526 (2005).

[23] H. J. Jensen, Adv. Solid State Phys. 45, 95 (2006).

[24] S. C. Srivastava and A. Lakshminarayan, Chaos Solitons Fractals 74, 67 (2015).

[25] Z. Danku and F. Kun, Front. Phys. 2, 8 (2014).

[26] J. Baró, A. Corral, X. Illa, A. Planes, E. K. H. Salje, W. Schranz, D. E. Soto-Parra, and E. Vives, Phys. Rev. Lett. 110, 088702 (2013).

[27] S. Lennartz-Sassinek, I. G. Main, M. Zaiser, and C. C. Graham, Phys. Rev. E 90, 052401 (2014).

[28] I. G. Main, Geophys. J. Int. 142, 151 (2000).

[29] M. Heap, P. Baud, P. Meredith, S. Vinciguerra, A. Bell, and I. Main, Earth Planet. Sci. Lett. 307, 71 (2011).

[30] I. G. Main, O. Kwon, B. T. Ngwenya, and S. C. Elphick, Geology 28, 1131 (2000).

[31] MATLAB, version 7.10.0 (R2010a) (The MathWorks Inc., Natick, Massachusetts, 2010).

[32] P. W. Miller and E. Ben-Naim, J. Stat. Mech.: Theor. Exp. (2013) P10025.

[33] C. H. Scholz, Bull. Seismol. Soc. Am. 58, 399 (1968).

[34] S. Redner and M. R. Petersen, Phys. Rev. E 74, 061114 (2006).

[35] J. Franke, G. Wergen, and J. Krug, Phys. Rev. Lett. 108, 064101 (2012). 